\title{
Adoption of Computed Tomography Images under Iterative Reconstruction Algorithm in Diagnosis of Gastric Cancer
}

\author{
Yan Chen $(\mathbb{D}$, Changkun Lin $(\mathbb{D}$, Qimao Fu $\mathbb{D}$, Jinghui Huang $\mathbb{D}$, and Chuizhi Huang $(\mathbb{D}$ \\ Department of Radiology, The Second Affiliated Hospital of Hainan Medical University, Haikou 570311, Hainan, China \\ Correspondence should be addressed to Chuizhi Huang; huangchuizhi@hainmc.edu.cn
}

Received 7 May 2021; Revised 3 June 2021; Accepted 11 July 2021; Published 20 July 2021

Academic Editor: Gustavo Ramirez

Copyright (c) 2021 Yan Chen et al. This is an open access article distributed under the Creative Commons Attribution License, which permits unrestricted use, distribution, and reproduction in any medium, provided the original work is properly cited.

\begin{abstract}
Objective. This work aimed to study the application of iterative reconstruction algorithm-based computed tomography (CT) imaging in the diagnosis of gastric cancer (GC). Methods. 40 cases of GC patients diagnosed by gastroscopy biopsy and pathology in hospital were retrospectively analyzed. Scanning images of the upper abdomen were obtained after plain scanning and doublephase enhanced scanning. Then, the image was reconstructed by the iterative reconstruction algorithm, and the CT value under the algorithm was analyzed statistically. Results. It was revealed that the detection rate of both spiral CT and iterative reconstruction algorithm-based CT was $100 \%$. After the iterative reconstruction algorithm, the image quality, image information, and image mean square error (MSE) were notably improved. The degree of tumor invasion (T) staging accuracy was $82.6 \%$, lymph node metastasis $(\mathrm{N})$ staging accuracy was $73.2 \%$, and tumor node metastasis (TNM) staging accuracy was $79.1 \%$. The accuracy of the iterative reconstruction algorithm-based CT was $90 \%$ for T staging, $83 \%$ for $\mathrm{N}$ staging, and $85.5 \%$ for TNM staging. Conclusion . Iterative reconstruction algorithm can effectively improve the spatial resolution of CT images in GC diagnosis, with high accuracy. It can provide reliable and objective imaging data for the diagnosis of GC clinically, which was worthy of further application in clinical practice.
\end{abstract}

\section{Introduction}

GC is one of the most common malignancies in the world, ranking the $2^{\text {nd }}$ in the line of death of malignancies. Asia, Japan, South Korea, and China are high incidence areas of GC, and more than half of the global GC patients are from East Asia [1]. Studies suggested that the occurrence and progression of GC is closely related to precancerous lesions [2]. Preoperative CT examination of GC can stage lymph node transfer, but the criteria and accuracy are still controversial, and the criteria for lymph node metastasis are not unified at present [3]. Studies also found that the occurrence and phylogeny of GC are a multistage and multistep process, involving the activation of multiple protooncogenes, deletion of tumor suppressor genes, or abnormalities in molecular biology [4]. Despite advances in surgery and multidisciplinary treatment, the prognosis of GC patients is still poor; especially for patients with advanced GC, the median survival time is only $7.5-12$ hours. Therefore, there is an urgent need for new therapies to improve the prognosis and survival time of GC patients.

Surgical resection is the most important treatment for $\mathrm{GC}$ at present, and accurate preoperative clinical staging is of great value and significance for selecting reasonable surgical methods, guiding treatment, and evaluating prognosis. Preoperative clinical staging included the extent of tumor invasion $(\mathrm{T})$, lymph node metastasis $(\mathrm{N})$, and distant metastasis [5]. The main imaging examinations in clinical staging included computed tomography (CT) and endoscopic ultrasound (EUS) [6]. CT imaging technology has been widely adopted in medicine, industry, and other fields. In the actual CT scanning process, due to the limited size of the detector or the large size of the object, the projection data projected on the detector is truncated, and the reconstructed CT image can only reflect part of the object information. Spiral CT is widely utilized in the clinical staging of GC and is considered to be the best examination for the assessment of clinical staging at present. It has the ability to evaluate the 
degree of tumor invasion, lymphatic metastasis, and distant metastasis in a noninvasive manner $[7,8]$.

According to the International Commission on Radiological Protection (IRCP) research certification, getting a full-body CT scan increases a subject's risk of cancer from radiation by about 8 percent [9]. CT imaging is based on Bill's law; the more the projection data obtained on the detector, the better the reconstruction of the image. The image is reconstructed based on the iterative reconstruction algorithm, and the image is clear, which can clearly show the location of the lesion. Therefore, it needs to keep the clinical $\mathrm{X}$-ray dose as low as possible. However, it will inevitably result in incomplete projection data, resulting in stripe artifacts in the reconstructed image and blurred image details. In addition, in the industry, due to some practical constraints, the scanning angle range becomes smaller. In this case, the reconstructed image will have serious artifacts in some directions. Based on the original equipment without affecting the normal diagnosis, how to effectively reduce the radiation dose of the patients, improve the image resolution, and bring accurate diagnosis for doctors have become the major issue facing the imaging industry [10]. Iterative reconstruction algorithm is a new generation of IR technique, which can obtain images in accordance with diagnostic requirements under low scanning conditions. Iterative algorithm is considered to be a better reconstruction method for incomplete projection and projection data with noise, although it has a large amount of computation and takes a long time. The optimization of CT reconstruction algorithm takes advantage of the original CT data acquisition. It can greatly reduce the noise on the image and improve the signal-to-noise ratio through repeated mathematical and physical operations, thereby reducing the radiation dose received by patients, and improving the image quality, spatial resolution, and density resolution of the image [11]. Therefore, it is of great significance to study the efficient CT reconstruction algorithm, make full use of the advantages of the algorithm, and flexibly apply it to clinical practice.

In this work, to ensure the quality of CT reconstruction image, reconstruction algorithm was adopted to further improve the quality of low-dose CT reconstruction image. Analytic reconstruction and statistical iterative reconstruction are the most commonly adopted methods for processing CT reconstruction. Compared with analytic reconstruction algorithm, the iterative algorithm is more flexible in calculation. The original image can be reconstructed from only the projection data, so as to obtain more accurate GC diagnosis results.

\section{Materials and Methods}

2.1. Algorithm Principle. The projection process equation of $A \times A$ size two-dimensional image is as follows:

$$
D=\mathrm{QH}
$$

In (1), $H$ represents the image vector of size $A A \times 1, Q$ represents the projection coefficient matrix of $Y W \times A A, W$ represents the projection angle, $Y$ represents the maximum number of projections at each projection angle, and $D$ represents the projection vector of size $Y W \times 1$.

The reconstructed image is shown in the following equation:

$$
B^{*}=Q^{*} D \text {. }
$$

In (2), $B^{*}$ is the reconstructed image of size $A A \times 1$ and $Q^{*}$ represents the generalized inverse of the projection coefficient matrix $Q$. The direct calculation of $Q^{*}$ will be slightly complicated and consume reconstruction time. It can be replaced by other calculations, which can save computing time.

According to the following principles, the first-order iterative method is adopted to obtain $Q^{*}$. If the initial estimate of the generalized inverse $Q^{*}$ of the projection coefficient matrix $Q$ of $Y W \times A A$ is set to $N_{0}$, the residual $S_{0}=D_{R(T)}-Q N_{0}$ is set to meet $\gamma S_{0}<1$, where $\gamma S_{0}$ represents the spectral radius of $S_{0}$, and $D_{R}(Q)$ is the orthogonal matrix of $Q$. Then, the sequence $\left[N_{0}, N_{1}, \ldots N_{K}, N_{K+1}, \ldots\right]$ can be expressed by the following equation:

$$
N_{K+1}=N_{k}+N_{0}-N_{0} Q N_{K}, \quad K=0,1, \ldots
$$

When $K \longrightarrow \infty$, the equation converges to $Q^{*}$, and then the corresponding sequence of the residual value meets the requirements of the following equation:

$$
\left\|S_{K+1}\right\| \leq\left\|S_{0}\right\| S_{K}, \quad K=0,1, \ldots
$$

The norm of any multiplication matrix conforms to $S_{K}=D_{R(T)}-Q N_{K}$.

For convenience, the approximate value $N_{0}$ of the initial value of $Q^{*}$ is simply considered to be equal to

$$
N_{0}=\theta Q^{t}
$$

In (5), $Q^{t}$ is the transposition of $Q$ and $\theta$ is an actual value, which conforms to

$$
0<\theta<\frac{2}{\lambda_{1}\left(Q Q^{t}\right)} .
$$

In (6), $\lambda_{1}\left(Q Q^{t}\right)$ is the largest nonzero eigenvalue of $Q Q^{t}$.

To avoid the difficulty of calculating the relatively large values of $Q$ and $Q^{q}$, both sides of (3) are multiplied by the projection value $D$ at the same time, and (7) is obtained:

$$
N_{K+1} D=N_{K} D+N_{0} D-N_{0} Q N_{K} D, \quad K=0,1, \ldots
$$

$N_{K+1} D$ and $N_{K} D$ represent the $(K+1)$-th and $K$-th reconstructed images $H_{K+1}^{i}$ and $H_{K}^{i}$, respectively. $N_{0} D$ is the initial image $H_{0}^{i} . N_{0} Q N_{K} D$ represents the projection of $H^{i}$. Finally, the reconstruction is performed. In this work, FBP algorithm is adopted to reconstruct the image $\theta$ times to replace $N_{0} Q N_{K} D$. When $A A<Y W, \theta=1$; when $N N>Y W$, $\theta<2^{-(A A / Y W)}$. Equation (7) can be simplified as follows:

$$
H_{K+1}^{i}=H_{K}^{i}+H_{0}^{i}-N_{0} Q H_{K}^{i} \text {. }
$$

To verify the performance of the algorithm, the following experiments are carried out. In the experiments, the value of $\theta$ is 1 . 
The basic principle of the iterative reconstruction algorithm for image processing is shown in Figure 1.

2.2. Algorithm Steps. According to the principle of the algorithm, the steps of the algorithm as shown in Figure 2 are as follows. In the first step, initialize $\theta$ and termination conditions $\varphi$. In the second step, when $K=0$, FBP algorithm reconstructs the image $H_{f b p}^{i}$ to obtain the preprocessed image $H_{0}^{i}=\theta H_{f l p}^{i}$. In the third step, project $H_{K}^{i}$ to obtain the projection value $D_{k}$. The fourth step is to reconstruct the image according to $D_{k}$ and FBP and multiply the result obtained by $\theta$ to obtain $H_{r}$. The fifth step is to correct the image. The equation is $H_{K+1}^{i}=H_{K}^{i}+H_{0}^{i}-H_{r}^{i}$. In the sixth step, set $\Delta=\left\|H_{K+1}^{i}-H_{K}^{i}\right\| \leq K=k+1$. In the last step, if $\Delta>\varphi$, it will return to step three; otherwise, the loop will end.

2.3. The Physical Basis ofCT Imaging. X-ray is a way for CT to obtain information about the internal structure of the human body. The most important way is to adopt a detector to capture the attenuated X-rays passing through the human body. The acquisition of the CT image is mainly to reconstruct the projection data acquired by the detector under different angles by the reconstruction of the algorithm.

For example, if the intensity of the $X$ tube generated by the device is set to $F_{0}$ and the weakened X-ray intensity after passing through the human body is set to $F_{\text {out }}$, then from Beer's law (the diagram of Beer's law is shown in Figure 3), the following equation is obtained:

$$
F_{\text {out }},=F_{0} \exp (-p)=F_{0} \exp (-\mu \mathrm{L}) \text {. }
$$

$P$ in (9) is the projection data obtained on the detector, $L$ represents the length of the X-ray passing through the object, and $\mu$ is the attenuation coefficient of the object to X-ray. In the medical field, the basic unit of CT reconstruction is generally voxels. Voxels divide the tissue into small blocks with uniform density, and the resulting uniform blocks are called voxels. The finer the division of the tissue, the smaller the voxel, indicating that the interval between the receivers on the detector is also smaller. For the same object, the detector can get more projection data, and the quality of the reconstructed image becomes better.

2.4. Basic Information. GC patients treated in our hospital were deemed as the research object. Inclusion criteria were the following: (1) those whose age is no less than 18 years; (2) patients diagnosed with GC after examination; (3) patients without other primary tumor diseases. Exclusion criteria were the following: (1) those with incomplete clinical data; (2) those who did not voluntarily participate in this research; (3) those who suffered from other diseases. According to the standard, a total of 40 cases were included. Twenty patients were selected as the CT group (including 11 males and 9 females), aged $45-70$ years, with an average of $(58.35 \pm 5.11)$ years. The remaining 20 cases were in the iterative algorithm CT group (Ira), including 12 males and 8 females, aged 45-70 years, with an average of $(59.35 \pm 5.09)$ years. All patients were confirmed to be GC by gastroscopy and were examined by

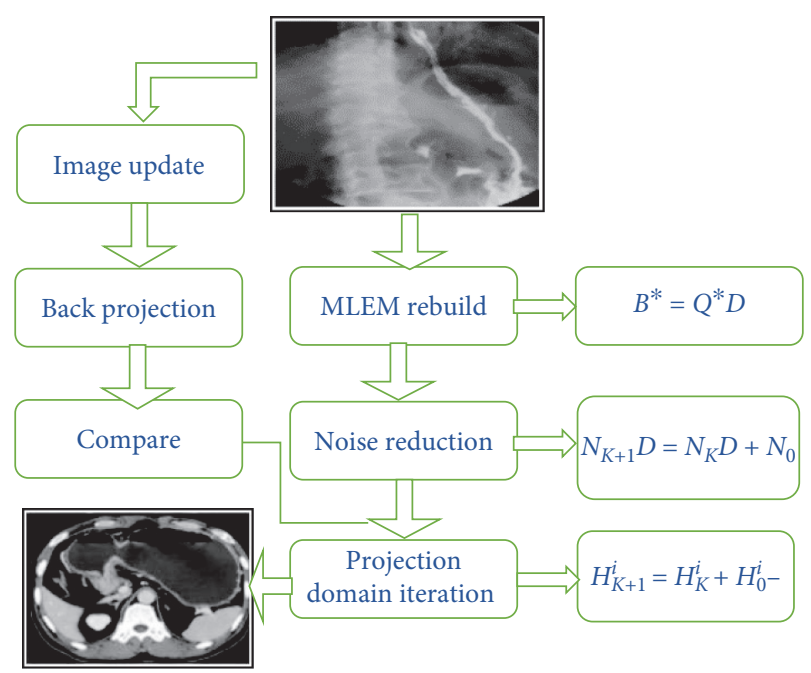

Figure 1: Flow chart of the principle of iterative reconstruction algorithm.

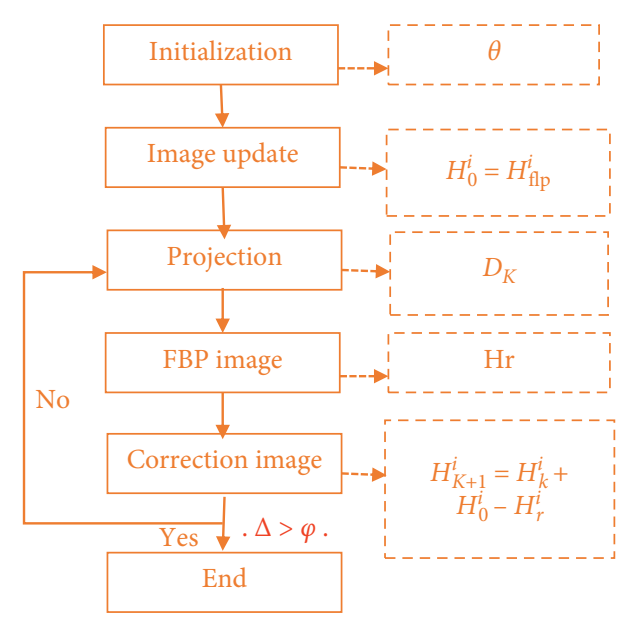

FIGURE 2: Flow chart of algorithm steps.

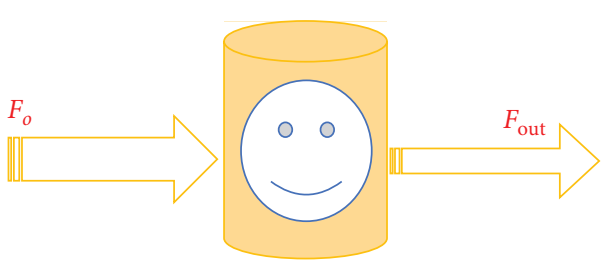

Figure 3: Schematic diagram of Beer's law.

multislice spiral CT within one week before surgery. Postoperative pathological examination was performed, and the results were compared with the results of the image reconstruction by the algorithm. This research was reviewed and approved by the hospital ethics committee. All the patients' family members had signed the informed consent.

2.5. Scanning Methods. All the patients involved in the research were maintained at fasting state before the 
examination and could drink a moderate amount of water before the examination. If patient had pyloric obstruction, it should first suck out the contents of the stomach. Before scanning, $15 \mathrm{mg}$ anisodamine was injected, and oral gasproducing powder was administered 20-30 min later. After inhalation, the breath was held. Philips 16-slice multislice spiral CT was employed to conduct the three-phase dynamic scanning of the patients. Scanning parameters were $110 \mathrm{kV}$, $180 \mathrm{mAs}$, pitch 0.8 , and layer thickness $5 \mathrm{~mm}$, and data collected was $16 \times 0.6 \mathrm{~mm}$. The arterial phase was from the xiphoid process to the umbilical cord and was performed at $75 \mathrm{~s}$ after contrast agent injection. The parenchymal phase was the scanning range of the whole stomach, which was carried out at $75 \mathrm{~s}$ after contrast agent injection. The balance phase was scanned from the diaphragmatic apex to the entire abdomen at $180 \mathrm{~s}$ after contrast agent injection. According to the patient's body weight, nonionic contrast agent $1.5 \mathrm{~mL} / \mathrm{kg}$ was injected intravenously from the elbow at a rate of $3 \mathrm{~mL} / \mathrm{s}$. The whole stomach was scanned by internal artery 20-35 s after injection. Combined with the scanning image results, a multiangle and multidirection were selected for careful observation and reconstruction of the image. The whole stomach was scanned from top to bottom, and the scanning results were recorded. All patients received the first MSCT scan within 5 days, and the first data were reconstructed by iterative algorithm, and the sensitive sites of the patients were protected during each scan.

2.6. Observation Indicators. When the conventional gastric wall thickness was greater than $5 \mathrm{~mm}$, CT scan would show multilayer structure. If the gastric wall was not consistent with the conventional gastric wall, GC can be determined. Clinically, many studies indicated that the length of peripheral gastric lymph nodes in multislice spiral CT images was greater than $6 \mathrm{~mm}$, and the length of peripheral gastric lymph nodes was greater than $8 \mathrm{~mm}$. According to the general TNM staging standard, $\mathrm{T}_{0}$ was the thickness of the stomach below $5 \mathrm{~mm}$ and had a one to three layers' structure. $\mathrm{T}_{1}$ : the monolayer gastric wall was thickened, and the submucosa had a complete low-density zone, and the fat layer around the stomach can be clearly seen. $\mathrm{T}_{2}$ : the thickened private density zone of the single gastric wall was destroyed or disappeared, the fat layer around the stomach and the outer surface of the stomach were clear and smooth, the multilayer gastric wall was enhanced, and the middle and outer layers suddenly disappeared. $\mathrm{T}_{3}$ : the fat layer around the stomach became microinfiltrated, the thickened gastric wall was irregular, or there was a cord-like high-density shadow on the outer boundary serosal surface. $\mathrm{T}_{4}$ : the whole layer of the gastric wall was spread by the lesion or encroaches on the tissues and organs around the stomach, and the infiltration of the fat layer in the gastric wall disappeared.

$\mathrm{N}$ staging took the Japanese GC Research Society "GC Processing Protocol 13 Edition" as the reference standard. N0 was no lymph node metastasis, and the distance and anatomical location of the primary tumor were three lymph node metastases. N1 was only transferred to the first station of the lymph node. N2 meant it was transferred to the second station of the lymph node. N3 meant it was transferred to the third station of the lymph node.

All image data were analyzed and compared by a very experienced attending physician and a senior physician. The reading content included CT image quality, position, and displacement and pathological analysis of the patient's T, N, and TNM staging.

2.7. Statistical Analysis. All data were processed by SPASS 21.0 software. Mean \pm standard deviation $(\bar{x} \pm s)$ was how measurement data were expressed. Pearson test was adopted for correlation analysis. When $P<0.05$, statistical differences were considered.

\section{Results}

3.1. Comparison of Pathological Analysis. Table 1 is the comparison of CT staging and pathological staging. The T, $\mathrm{N}$, and TNM values of GC patients had no correlation with pathological grade, and there was no considerable difference $(P>0.05)$. The number of patients in $\mathrm{N}$ stage was relatively large, while the number of patients in T stage and TNM stage was relatively small.

3.2. Iterative Clinical Images. In the case of using the same dose in Figure 4, the FBP algorithm-based image on the left had relatively large noise and strong graininess. The image on the right used iterative reconstruction algorithm, which greatly improved the image noise level and enhanced the smoothness of the image.

3.3. Reconstruction Result of Iterative Algorithm for Stomach CT Image. The iterative reconstruction algorithm reconstructed the image based on the estimation of the statistical model of the observation data. Figure 5(a) shows the imaging result of the iterative reconstruction algorithm, and Figure 5(b) on the right shows the marked part in Figure 5(a).

\subsection{Comparison of Image Results under Iterative Recon-} struction Algorithms. In Figure 6, the general image quality and image information of group $A$ reconstructed by the iterative algorithm were obviously superior to those of the spiral CT group. The iterative algorithm showed certain advantages in reconstructing the image.

\subsection{MSE of CT Image Based on Iterative Reconstruction} Algorithm. Figure 7 shows that, after a certain number of iterations, the iterative reconstruction algorithm can restore the original image. The closer the MSE was to zero, the better the effect was. The image quality after the reconstruction algorithm was relatively better.

3.6. Accuracy of T, N, and TNM. Figure 8 shows that the accuracies of the iterative reconstruction algorithm for CT images $\mathrm{T}, \mathrm{N}$, and $\mathrm{TNM}$ were $90 \%, 83 \%$, and $85.5 \%$, respectively, while those for spiral CT were $82.6 \%, 73.2 \%$, and 
TABLE 1: Comparison of CT staging and postoperative pathological staging.

\begin{tabular}{lcccc}
\hline & & T & T & TNM \\
& Age $(\bar{x} \pm s)$ years & $(1-2 / 3-4)$ & $(0-1 / 2-3)$ & 17 \\
$($ I-II/III-IV $)$ \\
\hline Iterative algorithms CT & $(58.35 \pm 5.11)$ & 2 & 12 & 7 \\
CT & $(58.35 \pm 5.11)$ & 1 & 0.68 & 0.76 \\
Correlation of association & 0.413 & 0.82 & $<0.001$ & $<0.001$ \\
$P$ & 0.623 & $<0.001$ & & 1 \\
\hline
\end{tabular}

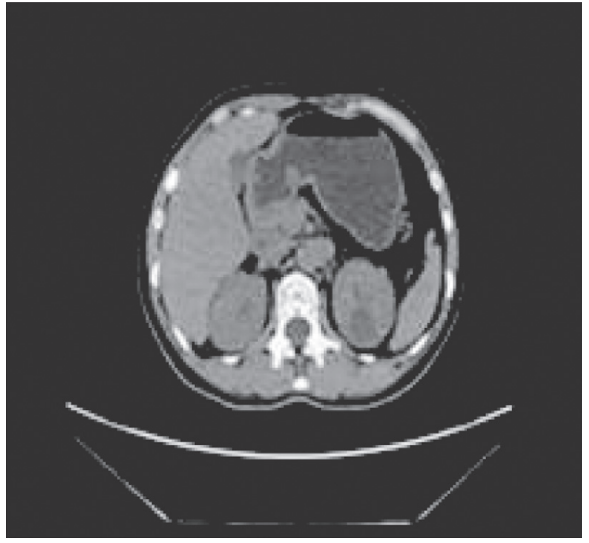

(a)

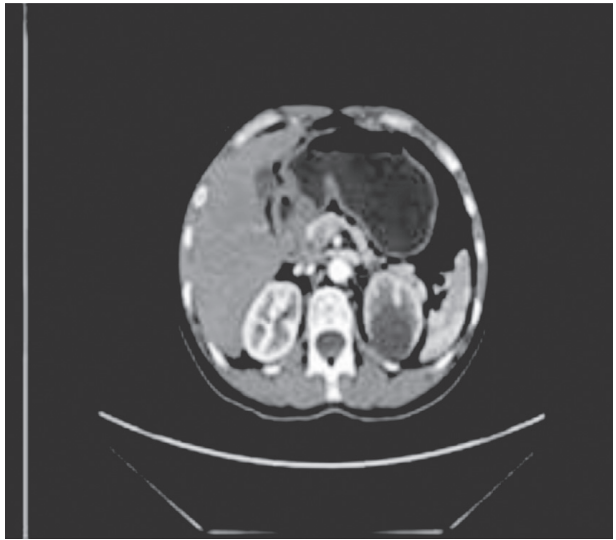

(b)

FIGURE 4: Comparison of low-dose original image of stomach and iterative reconstruction algorithm.

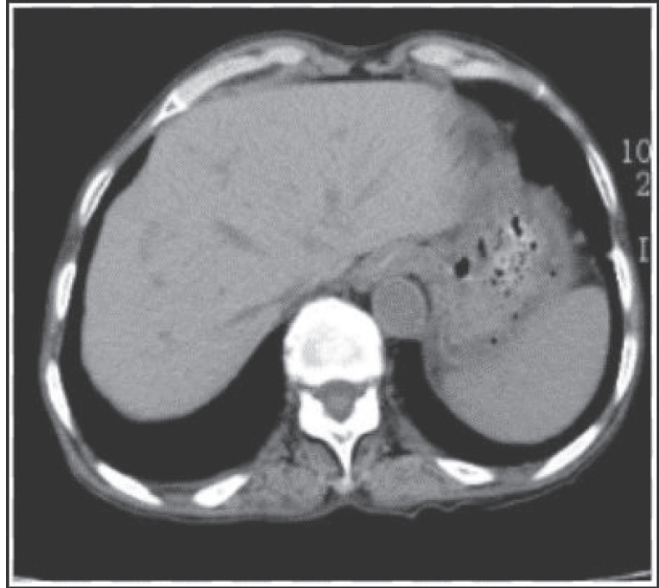

(a)

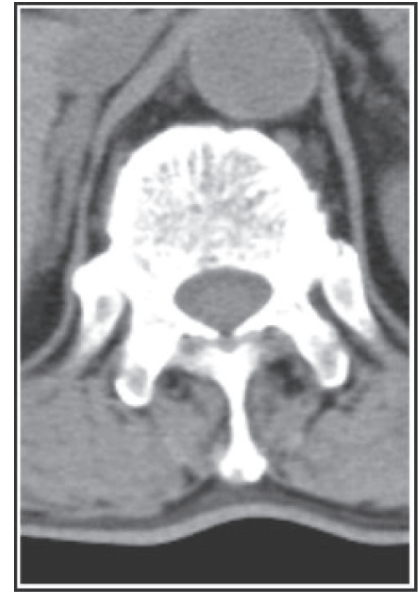

(b)

FIGURE 5: Iterative reconstruction algorithm results of the stomach. (a) Iterative reconstruction algorithm. (b) The marked portion of A.

$79.1 \%$, respectively. Therefore, the CT images under the iterative reconstruction algorithm were greatly superior to the spiral CT, indicating that the iterative algorithm CT reconstruction had relatively higher accuracy in diagnosing GC.

\section{Discussion}

Compared with single-slice spiral CT, multislice spiral CT has multiple rows of detectors, which not only improves the scanning speed, but also reduces the rotation time to $0.5 \mathrm{~s}$. It can also obtain multilayer images during one revolution [12]. Due to fast volume scan, data can be collected continuously over a large area of the body in a short period of time, and the information obtained can also be increased. After computer processing, a variety of technology imaging is completed and the image quality is high. Simulation endoscopy is true, and the detection rate of smaller lesions and mucosal lesions is improved, based on which the image 


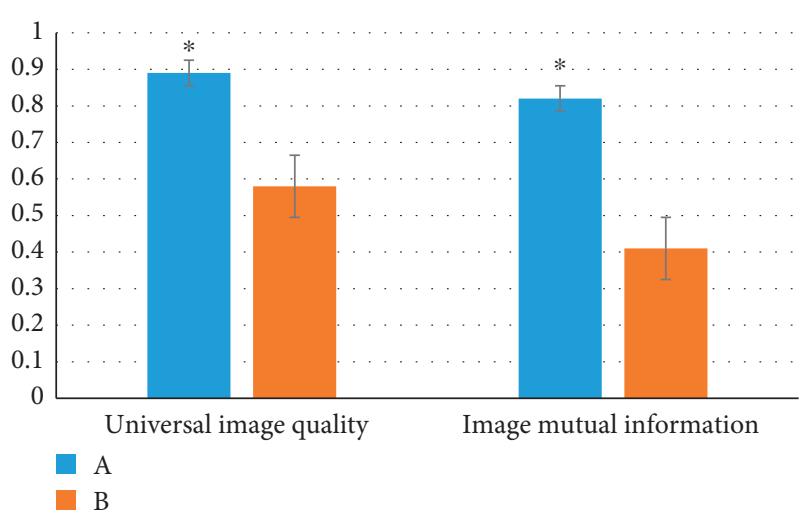

FIGURE 6: Image quality evaluation of iterative reconstruction algorithm (A: iterative reconstruction algorithm of CT group; B: spiral CT group) (* indicates statistically significant difference, $P<0.05)$.

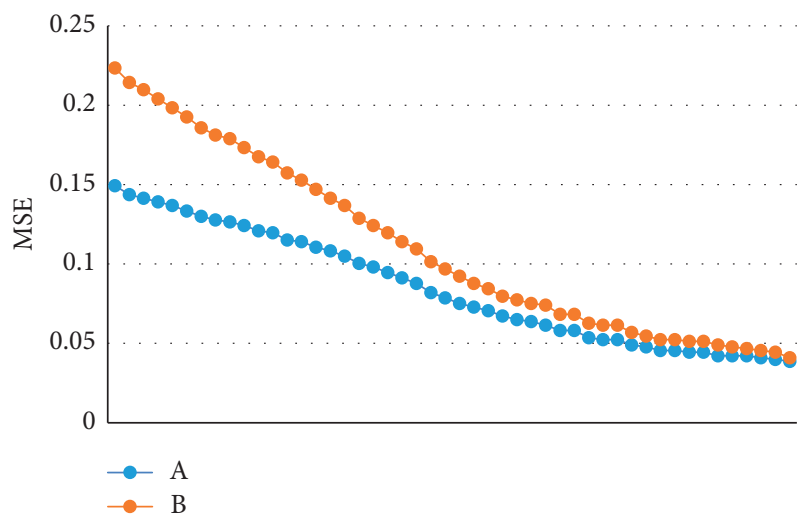

FIgURE 7: Image's MSE distribution map. (A: CT group without algorithm; B: CT group with iterative reconstruction algorithm).

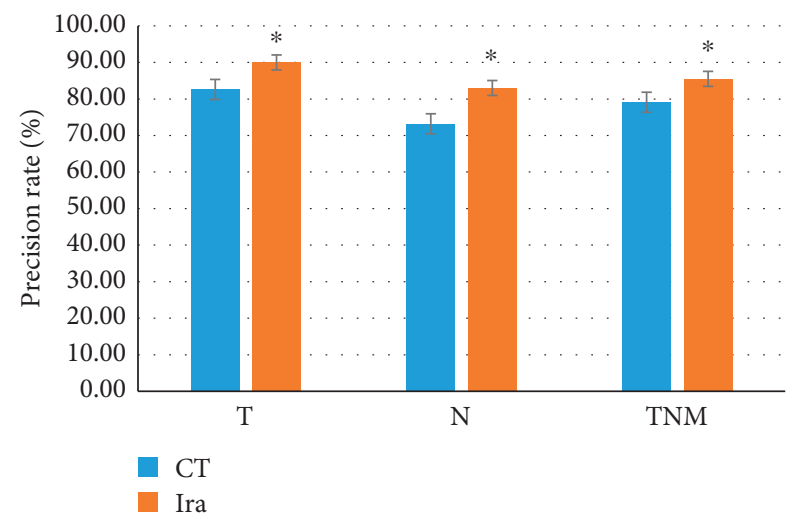

Figure 8: Comparison of accuracy of T, N, and TNM $\left({ }^{*}\right.$ indicates statistically significant difference, $P<0.05$ ).

quality can be improved effectively by iterative algorithm reconstruction. Some researchers utilized dual-source CT energy to scan the upper abdomen of GC patients under the condition of different tube voltages and employed different reconstruction algorithms to reconstruct the images under the CT dual-energy mode. The results showed that IRS technology can effectively reduce image noise [13], which was also the conclusion of this research. It has been reported that $28 \%$ of GC presented progressive enhancement from arterial to venous stage $[14,15]$. CT enhanced scan can show the relationship between the gastric wall layer, the lower layer of the membrane, and the muscular layer, which is helpful to judge the depth of GC infiltration. In this work, the gastric surface layer was not thoroughly studied, which gave a research direction for the next step.

The low-dose scanning was also adopted in this research, and the results showed that the iterative reconstruction algorithm had a detection rate of $100 \%$ in GCCT imaging scans. After optimization by the iterative reconstruction algorithm, the image quality, image information, and MSE of the image were all greatly improved. The closer the MSE was to zero, the smaller the error was and the more accurate the calculation result was. The accuracy of T staging of spiral CT was $82.6 \%$, the accuracy of N staging was $73.2 \%$, and the accuracy of TNM staging was $79.1 \%$. The accuracy of the iterative reconstruction algorithm was $90 \%$ for T staging, $83 \%$ for $\mathrm{N}$ staging, and $85.5 \%$ for TNM staging. The accuracy was considerably increased after reconstruction by the algorithm, which meant that the iterative reconstruction algorithm had relatively higher accuracy.

The diagnosis of TNM staging by GC CT is mainly based on the structure of the stomach wall, the contour of the serous membrane, that is, the changes in fat around the stomach, the invasion of the gastric-around organs, and the enlargement of lymph nodes. Tirumani et al. (2016) [16] believed that the accurate prediction of $\mathrm{T} 1$ stage can provide more useful prognostic information compared with the prognostic information of T3 stage, but for T1 GC patients, lymph node metastasis was very important, and expansion of lymph nodes would cause postoperative complications and mortality. Therefore, the iterative algorithm CT can provide more effective treatment for this type of patients. Some studies also deemed that CT tumor volume was significantly related to pathological $\mathrm{T}$ and $\mathrm{N}$ staging. In this research, there were fewer patients in T1 stage compared with T3 stage, which was consistent with the results of many literatures. It may be because, in the same time period, there were more GC patients at T3 stage. The low number of early GC cases may be related to the popularity of GC screening by the Chinese people and the attention of the public.

\section{Conclusion}

In this study, patients with gastric cancer were retrospectively analyzed, and the CT images of patients were analyzed and compared with the reconstructed images based on the iterative algorithm. The results showed that the accuracy of the iterative algorithm was $85.5 \%$, and the accuracy of CT was $82.6 \%$. Iterative algorithm was better than CT image in image staging accuracy. The quality of CT images was improved after optimization by the iterative reconstruction algorithm. The visual field was clear and intuitive, the resolution was also enhanced, and the operation steps were simple. These advantages effectively improved the traditional CT diagnosis of GC and laid the foundation for the 
quantitative analysis of GC in the future. The only shortcoming of this work is that the number of cases is not large, and the number of subjects included in the experiment is limited. At a certain time, it will be combined with multiple centers to conduct experiments.

\section{Data Availability}

No data were used to support this study.

\section{Conflicts of Interest}

The authors declare that they have no conflicts of interest.

\section{Authors' Contributions}

Yan Chen and Changkun Lin contributed equally to this work.

\section{References}

[1] E. C. Smyth, M. Nilsson, H. I. Grabsch, N. C. Van Grieken, and F. Lordick, "Gastric cancer," The Lancet, vol. 396, no. 10251, pp. 635-648, 2020.

[2] H. C. Lee, B. Song, J. S. Kim et al., "An efficient iterative CBCT reconstruction approach using gradient projection sparse reconstruction algorithm," Oncotarget, vol. 7, no. 52, pp. 87342-87350, 2016.

[3] A. Pryor, Y. Yang, A. Rana et al., "GENFIRE: a generalized Fourier iterative reconstruction algorithm for high-resolution 3D imaging," Scientific Reports, vol. 7, no. 1, Article ID 10409, 2017.

[4] K. H. Dekker, J. J. Battista, and K. J. Jordan, “Technical note: evaluation of an iterative reconstruction algorithm for optical CT radiation dosimetry," Medical Physics, vol. 44, no. 12, pp. 6678-6689, 2017.

[5] A. Omotayo and I. Elbakri, "Objective performance assessment of five computed tomography iterative reconstruction algorithms," Journal of X-Ray Science and Technology, vol. 24, no. 6, pp. 913-930, 2016.

[6] Z. Hou, J. Zhao, and J. Sun, "Inner focus iterative reconstruction method with the interlaced phase stepping scanning for grating-based phase contrast tomography," in Proceedings of the 2019 41st Annual International Conference of the IEEE Engineering in Medicine and Biology Society (EMBC), Berlin, Germany, July 2019.

[7] J. Cheng, J. Wu, N. Hong, and Y. Wang, "[Analysis of associated factors of extramural venous invasion detected by CT scan in gastric cancer]," Zhonghua Wei Chang Wai Ke Za Zhi, vol. 19, no. 3, pp. 300-303, 2016, in Chinese.

[8] Y. Wang, W. Liu, Y. Yu et al., "CT radiomics nomogram for the preoperative prediction of lymph node metastasis in gastric cancer," European Radiology, vol. 30, no. 2, pp. 976-986, 2020.

[9] S. Chang, M. Li, H. Yu et al., "Spectrum estimation-guided iterative reconstruction algorithm for dual energy CT, IEEE Transactions on Medical Imaging, vol. 39, no. 1, pp. 246-258, 2020.

[10] E. C. Gertsen, C. De Jongh, H. J. F. Brenkman et al., "The additive value of restaging-CT during neoadjuvant chemotherapy for gastric cancer," European Journal of Surgical Oncology, vol. 46, no. 7, pp. 1247-1253, 2020.
[11] J. Li, Y. Tan, D. Zhang et al., "Value and necessity of pelvic CT in gastric cancer staging: an observational study," Scandinavian Journal of Gastroenterology, vol. 53, no. 9, pp. 10971099, 2018.

[12] G. Sun, C. Cheng, X. Li, T. Wang, J. Yang, and D. Li, "Metabolic tumor burden on postsurgical PET/CT predicts survival of patients with gastric cancer," Cancer Imaging, vol. 19, no. 1, p. 18, 2019.

[13] D. Ichikawa, S. Komatsu, T Kosuga et al., "Clinicopathological characteristics of clinical early gastric cancer in the upperthird stomach," World Journal of Gastroenterology, vol. 21, no. 45, pp. 12851-12856, 2015.

[14] S. Liu, H. Shi, C. Ji et al., "Preoperative CT texture analysis of gastric cancer: correlations with postoperative TNM staging," Clinical Radiology, vol. 73, no. 8, pp. e1-756, 2018.

[15] M. Fairweather, K. Jajoo, N. Sainani, M. M. Bertagnolli, and J. Wang, "Accuracy of EUS and CT imaging in preoperative gastric cancer staging," Journal of Surgical Oncology, vol. 111, no. 8, pp. 1016-1020, 2015.

[16] S. H. Tirumani, A. B. Shinagare, A. C. O’Neill, M. Nishino, M. H. Rosenthal, and N. H. Ramaiya, "Accuracy and feasibility of estimated tumour volumetry in primary gastric gastrointestinal stromal tumours: validation using semiautomated technique in 127 patients," European Radiology, vol. 26, no. 1, pp. 286-295, 2016. 